\title{
Micorrización en un cultivo de papa adicionado del biofertilizante Twin-N establecido en un Andisol de la Región de La Araucanía
}

\author{
Mycorrhizae in a potato crop added Twin-N biofertilizer in an Andisol \\ of the Araucanía Region
}

\author{
Claudia Castillo $^{1 *}$, María José Huenchuleo ${ }^{1}$, Andrés Michaud², Jaime Solano ${ }^{1}$
}

\section{RESUMEN}

Los efectos negativos causados por los fertilizantes químicos al medio ambiente han conducido en los últimos años a la investigación de alternativas ecológicas para el manejo de los cultivos, como los biofertilizantes, que utilizan microorganismos fijadores de nitrógeno (N). Durante la temporada 2013-2014 se estableció un ensayo de campo en la Estación Experimental Pillanlelbún, que tuvo como objetivo evaluar el efecto del biofertilizante Twin-N sobre los hongos micorrícicos arbusculares (HMA) nativos en

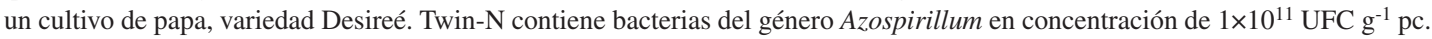
Se utilizó un diseño experimental de bloques completamente al azar con tres tratamientos y cuatro repeticiones. Los tratamientos correspondieron a: T0: un control con fertilización química a la siembra, FQS; T1 $=\mathrm{FQS}+50 \mathrm{~kg} \mathrm{de} \mathrm{N} \mathrm{ha}^{-1}$ (aporca) y T2 $=\mathrm{FQS}$ + $1 \mathrm{~L}$ Twin- $\mathrm{N} \mathrm{ha}^{-1}$ (floración). A la cosecha se cuantificaron parámetros fúngicos y número, rendimiento y condición sanitaria de los tubérculos. En relación con los parámetros HMA, el tratamiento con Twin-N presentó la menor colonización fúngica en las raíces y una baja cantidad de esporas en la rizósfera, acompañado de un significativo incremento en el rendimiento de los tubérculos, señalando que los microorganismos contenidos en el biofertilizante contribuyeron a mejorar la efectividad de los hongos nativos. La incidencia de enfermedades en los tubérculos fue baja, solo se encontraron diferencias en la pudrición seca y cráteres. Se concluye que la adición del biofertilizante Twin-N a un cultivo de papa podría reemplazar en parte la fertilización química nitrogenada disminuyendo los problemas de toxicidad en el suelo.

Palabras clave: hongos micorrícicos arbusculares, calibre del tubérculo, biofertilizantes, sarna en papas.

\begin{abstract}
Environmental concerns about negative effects caused by chemical fertilizers stimulated research on ecological crop management methods, in recent years, such as the use of biofertilizers, for example free living nitrogen-fixing microorganism. During the 20132014 seasons, a field trial was established at the Estación Experimental Pillanlelbún that aimed to assess the effect of the biofertilizer Twin $N$ on potato variety Desireé. Twin- $N$ contains bacterias of Azospirillum genera in a concentration of $1 \times 10^{11} U F C g^{-1} p c$. An experimental block design with completely randomized, three treatments and four repetitions was used. The treatments were: T0 = control with chemical fertilization at planting $(C F S), T 1=C F S+50 \mathrm{~kg} \mathrm{~N} \mathrm{ha}^{-1}$ (at ridging) and T2=CFS $+1 \mathrm{~L} \mathrm{Twin}-\mathrm{N} \mathrm{ha}^{-1}$ (at flowering). At harvest, number of tubers per plant, yield per hectare and health of tubers were recorded. Root colonization with arbuscular mycorrhizal fungi (AMF) was determined, and the number of spores of AMF was quantified. Regarding mycorrhizal fungal parameters Twin-N treatment had the lowest root colonization and a low number of spores in the rhizosphere; accompanied by a significant increase in tuber yield. Twin- $N$ apparently improved the effectiveness of native fungi. Differences were found in disease incidence of dry rot and craters. It is concluded that the addition of the biofertilizer Twin- $N$ to a potato crop can partly replace chemical nitrogen fertilization.
\end{abstract}

Key words: arbuscular mycorrhizal fungi, tuber size, biofertilizers, potato scab.

\section{Introducción}

A nivel mundial, el cultivo de papa constituye el cuarto alimento de mayor consumo en el mundo (Borda, 2008). En el país se cultivan anualmente alrededor de 50.000 ha con la mayor superficie concentrada en la Región de La Araucanía, cultivada por pequeños agricultores (ODEPA, 2015). El cultivo presenta serios problemas técnicos, entre ellos, la incidencia de hongos y bacterias en la

1 Escuela de Agronomía. Facultad de Recursos Naturales. Universidad Católica de Temuco. Rudecindo Ortega 02950. Temuco, Chile.

2 Empresa AMecological S.A.

* Autor para correspondencia: ccastill@uct.cl

Fecha de Recepción: 31 Agosto, 2015.

Fecha de Aceptación: 22 Diciembre, 2015. 
rizósfera que causan enfermedades en los tubérculos como rizoctoniasis, pudriciones y sarnas (Acuña y Santos, 2006). Para combatir estas enfermedades se recomienda el uso de variedades tolerantes y de tubérculos semilla saludables y certificados (Castro y Contreras, 2011). Una práctica agronómica que permite incrementar el rendimiento y calidad de los tubérculos es la fertilización química, ya que el cultivo requiere altas concentraciones de nitrógeno $(\mathrm{N})$, fósforo $(\mathrm{P})$ y potasio $(\mathrm{K})$, este último necesario en el proceso de tuberización (Inostroza, 2009).

Sin embargo, en la actualidad existe una preocupación creciente por el uso indiscriminado de productos químicos, orientándose la agricultura hacia un manejo más racional de los recursos disponibles (Alarcón y Ferrera, 2000), como la utilización de biofertilizantes, preparados de microorganismos, que mantienen el equilibrio biológico y físicoquímico de los suelos. Estos productos naturales pueden reemplazar a los sintéticos, reduciendo significativamente problemas de contaminación generados por el uso excesivo de agroquímicos (Armenta et al., 2010).

Algunos microorganismos utilizados en los biofertilizantes tienen la capacidad de sintetizar sustancias que promueven el crecimiento de la planta fijando $\mathrm{N}$ atmosférico, solubilizando hierro $(\mathrm{Fe}) \mathrm{o}$ $\mathrm{P}$ inorgánico o mejorando la tolerancia al estrés por sequía, salinidad, metales tóxicos o exceso de pesticidas. Otros productos son capaces de disminuir o prevenir los efectos causados por patógenos (Lucy et al., 2004). Un grupo de microorganismos benéficos son los hongos micorrícicos arbusculares (HMA), biótrofos obligados, que forman asociaciones mutualísticas con un amplio rango de plantas hospederas, incluyendo importantes cultivos de interés agrícola (Preger et al., 2007). En los sistemas de agricultura sustentable el manejo de los HMA como biofertilizantes reduce o elimina el uso de fertilizantes tradicionales mejorando la productividad de los cultivos (Douds et al., 2007). Sin embargo, las prácticas de manejo agrícola pueden afectar la composición y diversidad de las comunidades HMA (Mathimaran et al., 2007) causando un efecto negativo en la asociación simbiótica (Gosling et al., 2006). Por otra parte, la abundancia fúngica se reduce por aplicación de fertilización fosforada, por la perturbación mecánica producida por la labranza del suelo y por los cambios de plantas hospederas en los sistemas de rotación de cultivos (Preger et al., 2007).
Los HMA, y también algunos microorganismos de la rizósfera, como bacterias diazotróficas (Azotobacter y Azospirillum) presentes en Twin- $\mathrm{N} \circledast$ (Aguirre et al., 2009), un biofertilizante utilizado para el cultivo de papa, establecen interacciones benéficas con las raíces de las plantas, siendo estas asociaciones planta-microorganismos rizosféricos importantes para el desarrollo del vegetal y el control de enfermedades (Jeffries et al., 2003). Las bacterias presentes en Twin- $\mathrm{N}$ fijan $\mathrm{N}$ atmosférico y lo transforman en amonio, estimulando el crecimiento de la planta (Postgate, 1998). Las bacterias no penetran el sistema radical de la planta siendo atraídas solo por un conjunto de exudados que actúan como fuente de carbono y energía aportando entre el $25 \%$ y $50 \%$ de las necesidades de $\mathrm{N}$ de los cultivos (Kannalyar, 1997).

De acuerdo con lo anterior, el objetivo del presente estudio fue evaluar el efecto del uso del biofertilizante Twin-N sobre los HMA, rendimiento y calidad sanitaria de tubérculos en un cultivo de papa (Solanum tuberosum L.) variedad Desireé establecido en un Andisol de la Región de La Araucanía.

\section{Materiales y Métodos}

Durante la temporada agrícola 2013/2014 se estableció el ensayo en un Andisol serie Temuco, en la Estación Experimental Pillanlelbún, perteneciente a la Universidad Católica de Temuco (38 39' 17,24”'S; $72^{\circ} 27 ’ 00,79$ ’O), Región de La Araucanía. El laboreo del suelo se realizó a mediados de octubre de 2013 y la plantación en noviembre, utilizándose una variedad de papa comercial Desireé, caracterizada por poseer una piel lisa de color rojo brillante, pulpa de color crema y producción de tubérculos ovales alargados. Para la siembra se usaron tubérculossemilla de calibre 45 a $55 \mathrm{~cm}$ que se desinfectaron con fludioxonilo $2,5 \% \mathrm{p} / \mathrm{v}$ en dosis de $1 \mathrm{~L} \mathrm{t}^{-1} \mathrm{de}$ semilla. El tamaño de las parcelas fue de $3 \mathrm{~m} \mathrm{x}$ 2,1 $\mathrm{m}$ con tres hileras que incluyeron 22 plantas, equivalente a una densidad de 45.000 plantas ha $^{-1}$, realizándose la siembra a una distancia entre hilera de $0,75 \mathrm{~m}$ y sobre hilera de $0,30 \mathrm{~m}$. A la siembra se aplicó una fertilización base correspondiente a una dosis de $1200 \mathrm{~kg} \mathrm{ha}^{-1}$ de la mezcla papa 11:30:11. Los tratamientos fueron tres de acuerdo al manejo: a) solo fertilización base (T0); b) fertilización base más $50 \mathrm{~kg}$ de $\mathrm{N}$ en forma de urea, a la aporca (T1), 
y c) fertilización base más $1 \mathrm{~L}$ Twin- $\mathrm{N} \mathrm{ha}^{-1}$, en floración (T2).

El Twin-N es un biofertilizante desarrollado a base de bacterias fijadoras de $\mathrm{N}$ liofilizadas, del género Azospirillum y otros diazótropos en concentración de $1 \times 10^{11} \mathrm{UFC} \mathrm{g}^{-1} \mathrm{pc}$, que suministran con una inoculación por temporada, entre 30 a $110 \mathrm{~kg} \mathrm{~N} \mathrm{ha}^{-1}$, dependiendo de las condiciones estacionales y la humedad del suelo.

Durante el ensayo se realizaron dos aporcas con aplicación al follaje del fungicida clorotalonil $72 \%$ $\mathrm{p} / \mathrm{v}\left(1 \mathrm{~L} \mathrm{ha}^{-1}\right)$ y manteniendo un riego por aspersión de $50 \mathrm{~mm}$ por cada riego. Después de siete meses de desarrollo del cultivo se realizó la cosecha en mayo de 2014, extrayendo, desde la hilera central de cada parcela, cinco plantas y cinco muestras de suelo rizosférico.

\section{Parámetros evaluados}

a) Colonización HMA en papa. En trozos de raíces lavados, de un centímetro de longitud, se cuantificó el porcentaje de colonización mediante tinción con azul de tripán al 0,05\% p/v (Phillips y Hayman, 1970), bajo lupa estereoscópica según el método del intercepto de líneas (Giovanetti y Mosse, 1980).

b) Número de esporas HMA. Las esporas se aislaron del suelo rizosférico según metodología de Brundrett et al. (1996), que consiste en hacer pasar $20 \mathrm{~g}$ de suelo con agua a través de distintos tamices de $460 \mu \mathrm{m}$ hasta $53 \mu \mathrm{m}$. El contenido de los dos últimos tamices (106 y $53 \mu \mathrm{m}$ ) se transfiere a un tubo de centrífuga, se añade sacarosa al $70 \%$ $\mathrm{p} / \mathrm{v}$, se centrifuga a $3000 \mathrm{rpm}$ y las esporas retenidas en el tamiz de malla menor, después de lavadas, se transfieren a una placa Doncaster para su recuento.

c) Tubérculos. En cada planta se cosecharon los tubérculos, se contabilizaron y se pesaron para determinar el rendimiento. Luego se clasificaron por calibre de acuerdo con su diámetro ecuatorial en $<28 \mathrm{~mm} ; 28$ a $35 \mathrm{~mm} ; 35$ a $45 \mathrm{~mm} ; 45$ a 55 $\mathrm{mm} ; 55$ a $65 \mathrm{~mm}$ y $>65 \mathrm{~mm}$.

d) Presencia de enfermedades. Las enfermedades se determinaron por observación de los tubérculos, según el porcentaje de piel cubierta con distintas enfermedades, comparándolas con la escala creada para evaluar cada enfermedad, cuyos rangos son: $0=$ sano; $1 \%$ del área dañada; $10 \%$ del área dañada; $25 \%$ del área dañada; $50 \%$ del área dañada y $>75 \%$ del área dañada. Así, para sarna negra se observó el porcentaje de piel cubierta por los esclerocios que genera el patógeno Rhizoctonia solani; para la sarna común se evaluaron las lesiones causadas en los tubérculos por la bacteria Streptomyces scabies y para la sarna plateada el característico brillo plateado producido en la superficie del tubérculo causado por Helminthosporium solani. En relación con la pudrición seca, la sintomatología más común se presenta en tubérculos, con pudrición de la semilla en el campo o pudrición seca en el almacenaje, siendo producida por hongos del género Fusarium, mientras que los cráteres los causan nematodos que infectan los tubérculos desarrollando agallas con apariencia verrugosa.

\section{Análisis estadístico}

El diseño experimental correspondió a bloques completamente al azar con cuatro repeticiones. Los resultados se sometieron a una prueba de normalidad y homogeneidad, y posteriormente se realizó un análisis de varianza utilizando ANDEVA de una vía y el test de comparación múltiple de Tukey (p $\leq 0,05$ ). Para el análisis estadístico de los resultados se utilizó el programa estadístico R-project para Windows versión 3.1.2.

\section{Resultados y Discusión}

A la cosecha, en el porcentaje de colonización HMA de las raíces de las plantas de papa se observaron diferencias significativas entre el control y T2 (Twin N), tratamiento que presentó la menor micorrización (Figura 1A). En general, los porcentajes fueron bajos, en comparación con otros cultivos tradicionales como los cereales (Montaño et al., 2001), aun cuando el tiempo (noviembremayo) fue suficiente para el establecimiento de una colonización mayor. La alta fertilización química aplicada al cultivo de papa podría explicar esta baja micorrización de las raíces; sin embargo, estos porcentajes fueron superiores a los informados por Cesaro et al. (2008) en agroecosistemas cultivados con papa en Italia, que fluctuaron entre 2,1 y 5,5\% y de los encontrados por Douds et al. (2007) en un ensayo en campo con S. tuberosum cv. "Superior", donde la colonización alcanzó valores entre 11 y $14 \%$. En general, otros estudios en este cultivo han reportado un rango variable de colonización en las raíces llegando a valores muy altos (70-80\%) en condiciones de escaso P inicial en el suelo (Black 

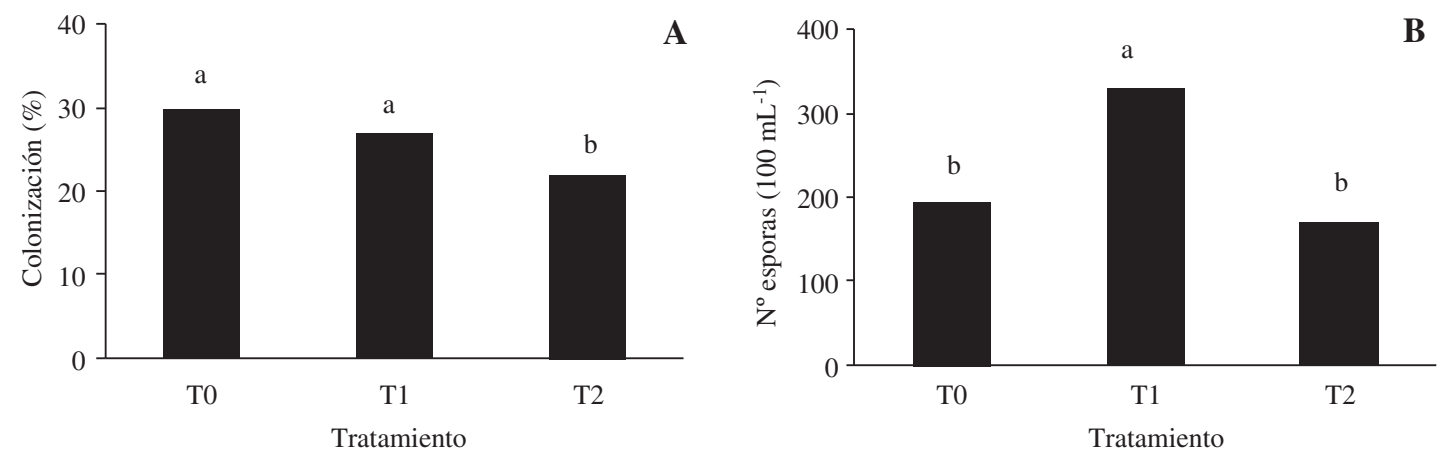

Figura 1. Parámetros fúngicos en raíces y rizósfera de papa variedad Desireé establecida en un Andisol de la Región de La Araucanía: A) Porcentaje colonización HMA y B) Número de esporas HMA. Barras seguidas de distinta letra presentan diferencias significativas según Test Tukey $(\mathrm{p} \leq 0,05)$.

y Tinker, 1977; Swaminathan y Verma, 1977; Montierro y Susana, 1983). McArthur y Knowles (1993) informaron que las condiciones fisiológicas podrían afectar la colonización fúngica de las raíces al observar que el porcentaje declina en el período de rápida tuberización, existiendo además diferencias en la susceptibilidad a la colonización entre cultivares de papa (Montierro y Susana, 1983).

Todas las esporas HMA se contabilizaron en este estudio, ya sea las jóvenes y las viejas. La mayor cantidad de esporas se cuantificó en el tratamiento con fertilización química (T1), presentando diferencias significativas respecto del control y T2 (Figura 1B); sin embargo, los rangos están dentro de los informados en la literatura que fluctúan desde 0,1 a 37 esporas $\mathrm{g}^{-1}$ suelo (Smith y Read, 1997). Los HMA mediante el micelio extrarradical transportan al sistema radical de las plantas, entre otros nutrientes, $\mathrm{P}$ y N, haciendo más eficiente la utilización de la fertilización química (Read, 1998), probablemente lo que ocurrió en el tratamiento T1. Según Davies et al. (2005), el número de esporas en el suelo es función, entre otros, de las especies HMA, clima, características del suelo y estacionalidad.

En relación con el rendimiento se encontraron diferencias entre T2 y T1. La aplicación del bioestimulante Twin- $\mathrm{N}$ incrementó de manera significativa el rendimiento del calibre consumo $(>6,5)$, con $36 \%$ sobre T1 (Figura 2). En consecuencia, resulta relevante que en el rendimiento total por hectárea el

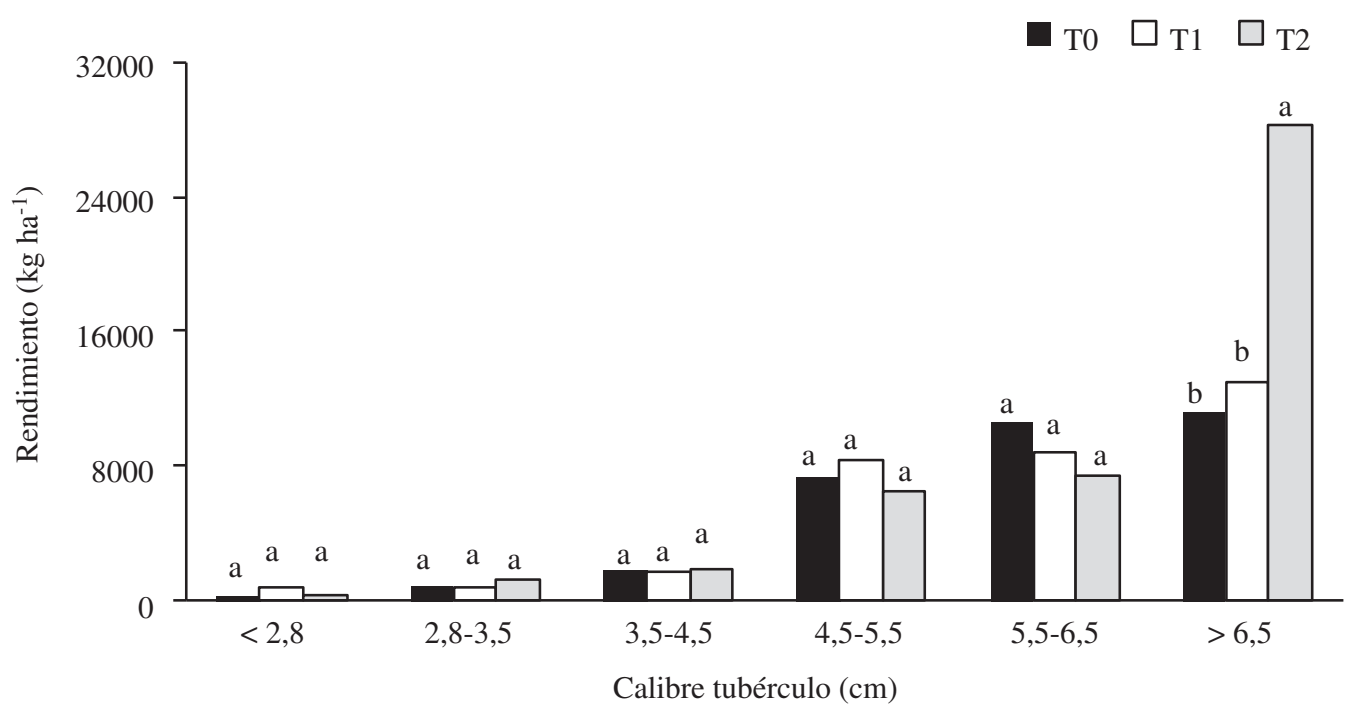

Figura 2. Rendimiento de acuerdo con la distribución de tubérculos por calibre de papa variedad Desireé establecida en un Andisol de la Región de La Araucanía. Barras seguidas de distinta letra presentan diferencias significativas según Test Tukey (p $\leq 0,05)$. 
tratamiento adicionado del biofertilizante fue superior en $116 \%$ en relación con el tratamiento de fertilización tradicional (Figura 3). Araujo et al. (2010) en un cultivo de papa usando un biofertilizante conteniendo Azotobacter sp., obtuvieron un rendimiento similar al del control con fertilización tradicional.

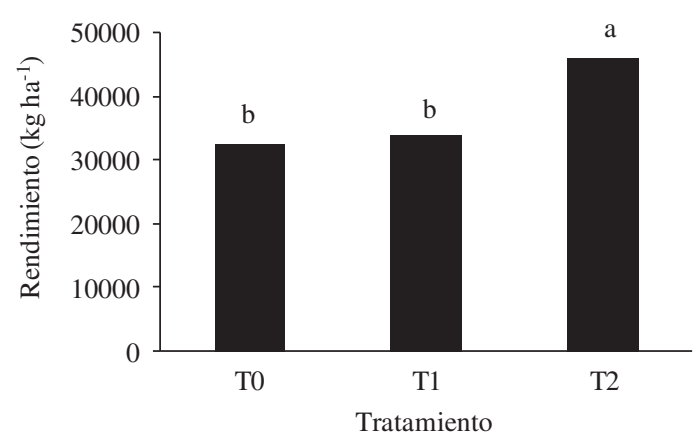

Figura 3. Rendimiento total $\left(\mathrm{kg} \mathrm{ha}^{-1}\right)$ de papa variedad Desireé establecida en un Andisol de la Región de La Araucanía. Barras seguidas de distinta letra presentan diferencias significativas según Test Tukey $(\mathrm{p} \leq 0,05)$.
En el número de tubérculos por calibre no se observaron diferencias entre los tratamientos, pero la aplicación de Twin- $\mathrm{N}$ en el calibre mayor a 6,5 aumentó al doble la cantidad (Tabla 1). Resultados similares obtuvo Glendinning (2008), quien con dos aplicaciones de Twin- $\mathrm{N}$ aumentó a más del doble el rendimiento de los tubérculos de mayor tamaño, entre 4,5 y $6,5 \mathrm{~cm}$. En general, el número de tubérculos por planta presentó una distribución homogénea, con un promedio de dos tubérculos por calibre y un total por planta de 13 (Tabla 1). Estos resultados son similares a los informados por Veitía et al. (2001), quienes contabilizaron un promedio de 14 tubérculos por planta.

Al momento de la cosecha, en relación con las condiciones sanitarias de los tubérculos, la mayoría se encontraban sanos (Tabla 2) con bajos niveles de incidencia de enfermedades, situándose principalmente entre $1 \%$ y $10 \%$ de lesiones en la piel del tubérculo.

Tabla 1. Distribución del número de tubérculos por calibre de papa variedad Desireé establecida en un Andisol de la Región de La Araucanía.

\begin{tabular}{|c|c|c|c|c|c|c|c|}
\hline \multirow{2}{*}{ Tratamiento } & \multicolumn{6}{|c|}{ Distribución de números de tubérculos por calibre } & \multirow{2}{*}{$\begin{array}{c}\mathrm{N}^{\circ} \text { tubérculos/ } \\
\text { planta }\end{array}$} \\
\hline & $<2,8$ & $2,8-3,5$ & $3,5-4,5$ & $4,5-5,5$ & $5,5-6,5$ & $>6,5$ & \\
\hline T 0 & 2 & 2 & 3 & 1 & 2 & 2 & 12 \\
\hline $\mathrm{T} 1$ & 1 & 2 & 3 & 2 & 1 & 4 & 13 \\
\hline $\mathrm{T} 2$ & 3 & 2 & 2 & 2 & 2 & 2 & 13 \\
\hline
\end{tabular}

Tabla 2. Incidencia (\%) de sarnas y otras enfermedades en los tubérculos cosechados de papa variedad Desireé establecida en un Andisol de la Región de La Araucanía.

\begin{tabular}{|c|c|c|c|c|c|c|c|}
\hline \multirow{2}{*}{ Enfermedad } & \multirow{2}{*}{ Tratamiento } & \multicolumn{6}{|c|}{ Incidencia $(\%)$} \\
\hline & & 0 & 1 & 10 & 25 & 50 & $>75$ \\
\hline \multirow[t]{3}{*}{ Sarna negra } & T0 & $52 \mathrm{a}$ & $44 \mathrm{a}$ & $4 \mathrm{a}$ & - & - & - \\
\hline & $\mathrm{T} 1$ & $41 \mathrm{a}$ & $52 \mathrm{a}$ & $6 a$ & $1 \mathrm{a}$ & - & - \\
\hline & $\mathrm{T} 2$ & $13 \mathrm{a}$ & $76 a$ & $9 \mathrm{a}$ & - & $2 \mathrm{a}$ & - \\
\hline \multirow[t]{3}{*}{ Sarna común } & T0 & $48 \mathrm{a}$ & $49 \mathrm{a}$ & $2 a$ & $1 \mathrm{a}$ & - & - \\
\hline & $\mathrm{T} 1$ & $41 \mathrm{a}$ & $46 a$ & $10 \mathrm{a}$ & - & $2 \mathrm{a}$ & $1 \mathrm{a}$ \\
\hline & $\mathrm{T} 2$ & $19 a$ & $71 \mathrm{a}$ & $8 \mathrm{a}$ & $1 \mathrm{a}$ & $1 \mathrm{a}$ & - \\
\hline \multirow[t]{3}{*}{ Sarna plateada } & T0 & $52 \mathrm{a}$ & $37 \mathrm{a}$ & $7 \mathrm{a}$ & $5 \mathrm{a}$ & - & - \\
\hline & $\mathrm{T} 1$ & $54 \mathrm{a}$ & $37 \mathrm{a}$ & $3 a$ & $6 a$ & - & - \\
\hline & $\mathrm{T} 2$ & $41 \mathrm{a}$ & $57 \mathrm{a}$ & $2 \mathrm{a}$ & - & - & - \\
\hline \multirow[t]{3}{*}{ Pudrición seca } & T0 & $99 a$ & - & - & $1 \mathrm{~b}$ & - & - \\
\hline & $\mathrm{T} 1$ & $95 \mathrm{a}$ & - & - & $5 \mathrm{a}$ & - & - \\
\hline & $\mathrm{T} 2$ & $99 a$ & - & - & $1 \mathrm{~b}$ & - & - \\
\hline \multirow{3}{*}{ Cráteres } & T0 & $94 \mathrm{a}$ & - & $5 \mathrm{a}$ & $1 \mathrm{~b}$ & - & - \\
\hline & $\mathrm{T} 1$ & $90 \mathrm{a}$ & - & $1 \mathrm{~b}$ & $9 \mathrm{ab}$ & - & - \\
\hline & $\mathrm{T} 2$ & $88 \mathrm{a}$ & - & $1 \mathrm{~b}$ & $11 \mathrm{a}$ & - & - \\
\hline
\end{tabular}

Letras distintas presentan diferencias estadísticamente significativas según test de Tukey $(\mathrm{p} \leq 0,05)$. 


\section{Conclusiones}

El cultivo de papa requiere grandes cantidades de recursos como agua, $\mathrm{N}$ y biocidas químicos. En sistemas de producción de cultivos sustentables, los hongos micorrícicos arbusculares junto con biofertilizantes como Twin-N, que incluyen en su composición bacterias fijadoras de $\mathrm{N}$, pueden ser una alternativa confiable de reemplazo parcial de fertilizantes químicos.

Finalmente, aumentar el conocimiento de la dinámica de las comunidades microbianas benéficas del suelo ofrece posibilidades de avance en la producción de papa.

\section{Literatura Citada}

Acuña, I.; Santos, J.

2006. Principales enfermedades del cultivo de papa. In: Manual de Producción de Papa para la Agricultura Familiar Campesina (AFC). Santos, J.; Orena, S. (eds.). Instituto de Investigaciones Agropecuarias, Osorno, Chile, pp. 115-132.

Aguirre, J.; Irizar, M.; Durán, A.; Grajeda, O.; Peña, M.; Loredo,

C.; Gutiérrez, Á.

2009. Los biofertilizantes microbianos: alternativa para la agricultura en México. Folleto Técnico $\mathrm{N}^{\circ} 5$, Instituto Nacional de Investigaciones Forestales, Agrícolas y Pecuarias, Tuxtla Chico, México, 80 pp.

Alarcón, A.; Ferrera, R.

2000. Biofertilizantes: importancia y utilización en la agricultura. Agricultura Técnica en México, 26 (2): 191-203.

Araujo, Y.; Díaz de García, L.; Rodríguez, F.; Pargas de González, L.

2010. Efecto del biofertilizante Azotobacter sp. en el cultivo de papa en el estado de Mérida. Derecho y Reforma Agraria Ambiente y Sociedad, 37: 17-33.

Armenta, B.A.D.; García, G.C.; Camacho, B.J.R.; Apodaca,

S.M.A.; Montoya, L.G.; Nava, P.E.

2010. Biofertilizantes en el desarrollo agrícola de México. Ra-Ximhai, 6: 51-56.

Black, R.L.B.; Tinker, P.B.

1997. Interaction between the effects of vesicular-arbuscular mycorrhizae and fertilizer phosphorus on yields of potatoes in the field. Nature, 267: 510-511.

Borda, N.

2008. La papa: un alimento básico. RAP-AL, Uruguay, 11 pp.

Brundrett, M.; Bougher, N.; Dell, B.; Grove, T.; Malajczuk, N. 1996. Working with mycorrhizas in forestry and agriculture. Australian Centre for International Agricultural Research (ACIAR), Canberra, Australia, 374 pp.

Castro, I.; Contreras, A.

2011. Manejo de plagas y enfermedades en el cultivo de la papa. Imprenta Austral, Valdivia, Chile, 72 pp.

Cesaro, P.; van Tuinen, D.; Copetta, A.; Chatagnier, O.; Berta,

G.; Gianinazzi, S.; Lingua, G.

2008. Preferential colonization of Solanum tuberosum L. roots by the fungus Glomus intraradices in arable soil of a potato farming area. Applied Environment Microbiology, 74: 5776-5783.

Davies, F.; Calderón, C.; Huaman, Z.

2005. Influence of arbuscular mycorrhizae indigenous to Perú and a flavonoid on growth, yield, and leaf elemental concentration of "Yungay" Potatoes. HortScience, 40 (2): 381-385.
Douds, D.; Nagahashi, G.; R.C.; Hepperly, P.

2007. Inoculation with arbuscular mycorrhizal fungi increases the yield of potatoes in a high P soil. Biological Agriculture and Horticulture, 25: 67-78.

Giovanetti, M.; Mosse, B.

1980. An evaluation of techniques for measuring vesiculararbuscular mycorrhizal infection in roots. New Phytologist, 84: 489-495.

Glendinning, $\mathrm{P}$.

2008. TwinN crop trial. Potato trial: Herefordshire, England $2007 \& 2008$. Available at http://www.amecological.com/ archivos/twin-n-trial-potato-trial-uk-2007-y-2008-lr.pdf. Accessed at 26 March 2015.

Gosling, P.; Hodge, A.; Goodlass, G.; Bending, G.D. 2006. Arbuscular mycorrhizal fungi and organic farming. Agriculture, Ecosystems and Environment, 113: 17-35.

Inostroza, J.

2009. Fertilización del cultivo de la papa. In Manual de papas en La Araucanía: manejo y plantación. Inostroza, J. (ed.). Instituto de Investigaciones Agropecuarias, Ministerio de Agricultura, Centro Regional Carillanca, Temuco, Chile, pp. 59-76.

Jefries, P.; Gianinazzi, S.; Perotto, S.; Turnau, K.K.; Barea, J.M. 2003. The contribution of arbuscular mycorrhizal fungi in sustainable maintenance of plant health and soil fertility. Biology and Fertility of Soils, 37: 1-16.

Kannalyar, S.

1997. The Indian experience for using biofertilizers. The Indian experience. In: Biological nitrogen fixation: the global challenge and future needs. Kennedy, I.R.; Cocking, E.C. (eds.). The Rockfeller Foundation Bellagio Conference Centre, Roma, Italy, pp. 69-70.

Lucy, M.; Reed, E.; Glick, B.R.

2004. Applications of free living plant growth-promoting rhizobacteria. Antonie Van Leeuwenhocka, 86: 1-25.

Mathimaran, N.; Ruh, R.; Jama, B.; Verchot, L.; Frossard, E.; Jansa, J.

2007. Impact of agricultural management on arbuscular mycorrhizal fungal communities in Kenyan ferralsol. Agriculture, Ecosystems and Environment, 119: 22-32.

McArthur, D.A J.; Knowles, N.R.

1993. Influence of vesicular-arbuscular mycorrhizal fungi on the response of potato to phosphorus deficiency. Plant Physiology, 101: 147-160.

Montaño, N.; Quiroz, V., Cruz, G.

2001. Colonización micorrícica arbuscular y fertilización mineral de genotipos de maíz y trigo cultivados en un Andisol. Terra Latinoamericana, 19 (4): 337-344. 
Montierro, C.G.; Susana, B.

1983. Response of a diverse group of thirty five cultivares to an insothermic environment of the Philipines. International Potato Center, Philippines, 8 pp.

ODEPA.

2015. Boletín de la papa, enero 2015. Disponible en http:// www.odepa.cl/wp-content/files_mf/1421413365boletin_ Papa201501.pdf. Consultado: 23/May/2015.

Phillips, J.; Hayman, D.

1970. Improved procedures for cleaning roots and staining parasitic and vesicular arbuscular mycorrhizal fungi for rapid assessment of infection. Transactions of the British Mycological Society, 55: 345-525.

Postgate, J.

1998. Nitrogen fixation. $3^{\text {rd }}$ edition. Cambridge University Press, Cambridge UK, 124 pp.

Preger, A.C.; Rilling, M.C.; Johns, A.R.; Preez, C.C.; Lobe, I.; Amelung, W.

2007. Losses of glomalin-related soil protein under prolonged arable cropping: A chronosequence study in sandy soils of the South African Highveld. Soil Biology and Biochemistry, 39: 445-453.

Read, D.

1998. Plants on the web. Nature 396: 22-23.

Smith, S.E.; Read, D.M.

1997. Mycorrhizal symbiosis. $2^{\text {nd }}$ ed. Academic Press, London, U.K., 605 pp.

Swaminathan, K.; Verma, B.C.

1977. Symbiotic effect of vesicular-arbuscular mycorrhizal fungi on the phosphate nutrition of potatoes. Proceeding of the Indian Academy of Sciences Section B, 85: 310-318.

Veitía, N.; Angel-Dita, M.; García, L.; Herrera, L.; Bermudez, I.; Acosta, M.; Clavero, J.; Orellana, P.; Romero, C.; García, L. 2001. The use of tissue culture and in vitro mutagenesis for the improvement of resistance to Alternaria solani in Irish Potato (Solanum tuberosum L.) var. Desiree. Biotechnology Vegetal, 1 (1): 43-48. 
